Research Paper

\title{
HTRAI Variants and the Interaction with Smoking Confer the Genetic Susceptibility to Ischemic Stroke
}

\author{
Yuanrui Tian ${ }^{*}$, Wuzhuang Tang ${ }^{2 *}$, Song Yang ${ }^{*}$, Yanping Zhao ${ }^{2}$, Yanchun Chen ${ }^{3}$, Xianghai Zhao ${ }^{3}$, Chunlan \\ Liu $^{1}$, Xiaotian Chen 1 , Chong Shen ${ }^{1 凶}$ \\ 1. Department of Epidemiology, School of Public Health, Nanjing Medical University, Nanjing 211166, China. \\ 2. Department of Neurology, Affiliated Yixing People's Hospital of Jiangsu University, People's Hospital of Yixing City, Yixing 214200, China. \\ 3. Department of Cardiology, Affiliated Yixing People's Hospital of Jiangsu University, People's Hospital of Yixing City, Yixing 214200, China. \\ *Yuanrui Tian, Wuzhuang Tang and Song Yang equally contributed to this work. \\ $\triangle$ Corresponding author: Chong Shen, Ph.D., Professor, Department of Epidemiology, School of Public Health, Nanjing Medical University, 101 Longmian \\ Avenue, Jiangning, Nanjing, 211166 China; Tel: +86 25 86868443; Fax: +86 25 86527613; E-mail: sc100@126.com \\ (c) The author(s). This is an open access article distributed under the terms of the Creative Commons Attribution License (https://creativecommons.org/licenses/by/4.0/). \\ See http://ivyspring.com/terms for full terms and conditions.
}

Received: 2020.03.10; Accepted: 2021.02.11; Published: 2021.02.23

\begin{abstract}
High temperature requirement protein Al ( $\mathrm{HtrAl})$ was identified as the causative gene of autosomal recessive arteriopathy and associated with lacunar ischemic stroke (IS) in European. This study aimed at evaluating the association of HTRAl with IS and four tagging single-nucleotide polymorphisms (SNPs) were genotyped in a cohort of 4,098 Chinese. The mRNA level of HTRAI in 72 IS cases and 72 hypertension controls were measured and compared. In whole population, SNP rs2268350 (C>T) was significantly associated with IS incidence $(P=0.034)$. Stratification analysis observed significant association of rs2268350 in male, smoking and drinking populations, rs2672587 (C>G) in smoking and nonsmoking populations and rs3793917 (C>G) in smoking, nonsmoking and nondrinking populations with stroke respectively $(P<0.05)$. The additive interaction and multiplicative interaction between $r s 2268350$ and smoking were both of significant $(P<0.05)$ after adjustment for the covariates. There was a cumulated risk of IS among genotypes of rs3793917 $(P=0.009)$ and rs2672587 $(P=0.047)$ in smoking population. The mRNA level of HTRAI in non-smokers with rs2268350 CC was significantly higher than smokers with rs2268350 CT/TT $(\mathrm{P}=0.046)$ in IS cases. Our findings support that HTRAl confers the genetic susceptibility to IS and smoking might modify the genetic effect of HTRAI on IS by suppressing HTRAI mRNA expression.
\end{abstract}

Key words: HTRA1; ischemic stroke; smoking; interaction; mRNA.

\section{Introduction}

The Global Burden of Disease 2016 Study (GBD 2016) has pointed out that 5.53 million people had different types of strokes worldwide in 2016 [1].

Stroke has become the second cause of death and major cause of disability in the world [2] while it's the leading cause of death in China [3]. In China, the remarkably increased number of ischemic stroke (IS) has resulted in 301 million disability-adjusted life-years [4].

Stroke including either ischemic or haemorrhagic stroke and about $80 \%$ of all strokes are ischemic [5]. The predisposing risk factors for stroke include hypertension (HTN), dyslipidemia, impaired glucose metabolism, metabolic obesity, smoking, lack of exercise and a family history of stroke [6,7]. In the pathophysiology of IS, the hazards of smoking on the risk of IS may partly be explained by changes in the venules [8], particularly, wider retinal venular caliber [9]. The number of cigarettes smoked daily show a strong dose-response relationship to the incidence of IS among young men [10], as well as, exposure to second-hand smoke can also significantly increase the risk of stroke [11].

The HtrA serine protease 1 (HTRA1) was the first one of the serine proteases family to be found $[12,13]$. HTRA1 represents diverse biological functions of 
vascular smooth muscle cell including growth, proliferation, migration, and apoptosis [14, 15]. HTRA1 inhibits transforming growth factor- $\beta$ (TGF- $\beta$ ) signal transduction by binding TGF- $\beta 1$, TGF- $\beta 2$, BMP2, BMP4, activin and Gdf5, thus inducing vascular changes [16-18]. In addition, HTRA1mediated proteolysis has been implicated in carcinogenesis [19], age-related macular degeneration (AMD) [20] and familial ischemic cerebral small-vessel disease [21]. The HTRA1-smoking additive effect was observed on AMD [22] and thus, the interaction between HTRA1 and smoking on cerebrovascular diseases is very noteworthy.

Hara et al have indicated that HtrA1 is the causative gene of Cerebral autosomal recessive arteriopathy with subcortical infarcts and leukoencephalopathy (CARASIL), a genetic cause of stroke in the young [21]. Recently, two rare monogenic variants were identified by target gene sequencing for younger onset lacunar stroke [23]. Patients with CARASIL are characterized by ischemic, non-hypertensive with associated alopecia and spondylosis [24-26]. A recent genome-wide association study (GWAS) [27] suggested that a single nucleotide polymorphisms (SNPs) rs79043147 in HTRA1 was associated with lacunar IS in individuals of European ancestry. However, the minor allele frequency (MAF) of SNP rs79043147 (C>T) is zero in Chinese population. Herein, it is necessary to evaluate whether HTRA1 harbor susceptible loci for IS in Chinese population.

This study focuses on the association of four tagging SNPs (tagSNPs) at HTRA1 with the incidence of IS in a cohort study of Chinese Han population and a population-based mRNA levels analysis which would help us well understand the effect of HTRA1 on IS.

\section{Method}

\section{Study participants}

A cluster sampling method was used to survey 4,128 subjects in 2009, Yixing city and 4,098 individuals without stroke were further followed up from 2014 to 2017. In addition, the mRNA was isolated from peripheral blood mononuclear cells (PBMCs) and compared between 72 IS patients from People's Hospital of Yixing City and age- ( \pm 2 years) and gender-matched 72 HTN controls from a community survey. IS cases included in mRNA analysis were diagnosed by CT and/or MRI examination that showed presence of infarction and categorized according to ICD-10 I60-I69.

The trained staff conducted a questionnaire survey to collect demographic data including age, gender, the habits of smoking and drinking and history of disease. The individuals who smoked more than 20 cigarettes per week for at least 3 months per year were defined as smokers. Drinking referred to the habit of currently or previously consuming alcoholic beverages more than 1 time per week for more than 5 months per year. Furthermore, weight $(\mathrm{kg})$ and height $(\mathrm{cm})$ were measured twice and the blood pressure level was averaged by the three times measurements [28].

All the subjects approved to donate $5 \mathrm{ml}$ venous blood. The serum glucose (GLU), total cholesterol (TC), triglyceride (TG), high-density lipoprotein cholesterol (HDL-C) and low-density lipoprotein cholesterol (LDL-C) levels were measured.

During the follow-up study, face to face interview and telephone interview were conducted to determine participants' disease incidence and vital data. The incident events were confirmed by the registered disease and verbal autopsy from the local public health authority (ICD-10 I60-I69) and IS events were finally determined by the study-wide endpoint assessment committee.

The study protocol was approved by the ethics committee of Nanjing Medical University (200803307, 2015077) and each participant signed a written informed consent.

\section{SNP Selection}

In the Han Chinese in Beijing (CHB), China, we searched all 84 SNPs with MAF over 0.05 covered HTRA1 gene with the addition of $2 \mathrm{~kb}$ upstream and 1 $\mathrm{kb}$ downstream to screen susceptible SNPs for IS. The tagSNPs were selected according to the criterion of linkage disequilibrium (LD) $\mathrm{r}^{2} \geq 0.8$, with the data from SNPinfo Web Server (http://snpinfo.niehs.nih.gov/). Finally, from 11 tagSNPs clusters and 16 single SNPs, we selected four tagSNPs in HTRA1, rs2268350 (C>T), rs2672587 (C>G), rs3793917 (C>G) and rs12413729 $(\mathrm{G}>\mathrm{A})$ with predicted biological function (Supplementary Table S1) that could tag 38 SNPs.

\section{DNA isolating and SNP genotyping}

A standard phenol-chloroform method was used to isolate the genomic DNA. The polymerase chain reaction (PCR)-TaqMan MGB probe array was performed to amplify all four tagSNPs of HTRA1 by GeneAmp ${ }^{\circledR}$ PCR system 9700 (Applied Biosystems, USA) thermal cycler and then read on the ABI 7900 system (Applied BioSystems, Foster City, CA). All the successful call rates of the four tagSNPs were over $99.79 \%$. 
Table 1. Baseline characteristics of the study population

\begin{tabular}{|c|c|c|}
\hline Characteristics & Group & Value \\
\hline $\mathrm{n}$ & & 4098 \\
\hline \multirow[t]{2}{*}{ Gender } & Male & $1661(40.5 \%)$ \\
\hline & Female & $2437(59.5 \%)$ \\
\hline Age (year) & & $59.20(52.75-67.00)$ \\
\hline \multirow[t]{2}{*}{ Blood pressure (mmHg) } & SBP & $134(123-141)$ \\
\hline & DBP & $82(78-89)$ \\
\hline Hypertension (\%) & & $1985(48.4 \%)$ \\
\hline $\mathrm{TC}(\mathrm{mmol} / \mathrm{L})$ & & $4.79(4.22-5.45)$ \\
\hline TG (mmol/L) & & $1.32(0.90-2.00)$ \\
\hline HDL-C (mmol/L) & & $1.34(1.14-1.56)$ \\
\hline LDL-C (mmol/L) & & $2.65(2.20-3.11)$ \\
\hline GLU (mmol/L) & & $5.28(4.85-5.80)$ \\
\hline T2DM (\%) & & $461(11.2 \%)$ \\
\hline BMI $\left(\mathrm{kg} / \mathrm{m}^{2}\right)$ & & $24.0(21.9,26.4)$ \\
\hline \multirow[t]{3}{*}{ BMI Group (\%) } & Normal (18.5-23.9) & $2047(50.0 \%)$ \\
\hline & Overweight (24-27.9) & $1521(37.1 \%)$ \\
\hline & Obesity $(>28)$ & $530(13.0 \%)$ \\
\hline \multirow[t]{2}{*}{ Smoking } & Yes & $995(24.3 \%)$ \\
\hline & No & $3103(75.7 \%)$ \\
\hline \multirow[t]{2}{*}{ Drinking } & Yes & $883(21.5 \%)$ \\
\hline & No & $3215(78.5)$ \\
\hline
\end{tabular}

\section{RNA Extraction and Real-time PCR detection}

After fasting over 12 hours, EDTA-containing blood samples of IS case were collected within 24 hours after admission in hospital and the samples of control were collected during the survey. Anticoagulant samples were immediately mixed with blood preservation solution (Eaglink Cat\#EGEN2026, NANJING YININGFUSHENG Biotech. Co., Ltd. Nanjing, China) by 1:3. The total RNA in PBMCs was isolated from $800 \mu \mathrm{l}$ mixture using RNA Blood Kit (Cat\#Yu-B02-1, Yuan Corp., Wuxi, China). cDNA was synthesized from mRNA using TAKARA reverse transcription kits (RR047A Takara PrimeScript RT reagent Kit with gDNA Eraser, Japan). The RT-PCR reactions were performed by ABI RT-PCR 7900. Each sample was prepared with three parallel samples and quality control requires that the standard deviation of cycle threshold (CT) among repeated samples is less than 0.5. The housekeeper gene of Glyceraldehyde-3-phosphate dehydrogenase (GAPDH) was used as reference gene for internal control. The mRNA relative expression is calculated by $2^{-\Delta \Delta \mathrm{CT}}(\Delta \Delta \mathrm{CT}$ case $=\Delta \mathrm{CT}$ case $-\Delta \mathrm{CT}$ control average value, $\Delta \Delta \mathrm{CT}$ control $=\Delta \mathrm{CT}$ control $-\Delta \mathrm{CT}$ control average value, $\Delta \mathrm{CT}=\mathrm{CT}$ of target gene - $\mathrm{CT}$ of housekeeper gene). All the reverse transcription and RT-PCR reactions as well as conditions are described at the Supplementary Information Reverse transcription reactions and conditions and RT-PCR reactions and conditions.

\section{Statistical analysis}

The questionnaire data was input by EpiData 3.0 software, IBM-SPSS 20.0was used for the statistical analysis. Cox regression model was used to estimate the association with hazard ratios (HRs) and 95\% confidence interval (CI). Mann-Whitney U test was applied to compare the mRNA levels with abnormal distribution between IS cases and controls. Statistical significance was defined as a two-tailed $P$ value less than 0.05. False discovery rate (FDR) method performed on R-software (V4.01) was used to correct multiple comparison [29]. Moreover, additive interaction was estimated by relative excess risk owing to interaction (RERI), attributable proportion (AP) owing to interaction and synergy index (S). An Excel sheet was used to calculate the additive interaction and their confidence intervals (www.epinet.se).

\section{Results}

\section{Demographic and clinical characteristics of study population}

The participants' demographic and clinical characteristics are listed in Table 1. During the 5.01 years median follow-up period, a total of 187 IS were finally observed by disease register and report system with an incidence density of 88.49 per $10^{4}$ person-years. Among the 187 IS, 12 IS (6.4\%) were less than 55 years old and 175 IS $(93.6 \%)$ were older than 55 years old. In addition, these 187 IS subjects consist of 94 men $(50.3 \%)$ and 92 women $(49.7 \%)$.

\section{Association analyses of HTRAI and IS incidence}

Cox regression analysis showed that the rs2268350 (C>T) TT genotype carriers had an increased risk of IS than CC and CT genotype carriers with a marginal $P$ value of 0.078 , the adjusted HR $(95 \% \mathrm{CI})$ was 1.499 (1.030-2.181) and $P$ value as 0.034 (Supplementary Table S2). Further stratification analysis revealed that in the male and smoking populations, the adjusted HRs $(95 \% \mathrm{CI})$ were 2.057 (1.240-3.411) and 2.933 (1.464-5.875) with $P$ values of 0.005 and 0.002 respectively. In smoking populations, the FDR-adjusted $P$ value was 0.048 . In drinking population, the genetic variants of rs2268350 presented an additive effect on the risk of IS and the HR (95\%CI) after adjustment was 1.855 (1.119-3.074) and $P$ value was 0.017 (Table 2).

Moreover, in the non-smoking population, the rs2672587 C>G variation was significantly associated with the increased risk of IS after adjustment for covariates, and $\mathrm{HR}(95 \% \mathrm{CI})$ of additive model was 1.662 (1.076-2.569), $P=0.022$. While in smoking population, the variants of rs2672587 presented an additive effect on the decreased risk of IS and the adjusted HR $(95 \% \mathrm{CI})$ was $0.631(0.405-0.985)$ with $P$ value of 0.043 (Table 2). 
Table 2. Stratification analyses of HTRAI polymorphisms and IS incidence in follow-up study

\begin{tabular}{|c|c|c|c|c|c|c|c|c|}
\hline \multirow[t]{2}{*}{ SNP } & \multirow[t]{2}{*}{ Stratum } & \multirow[t]{2}{*}{ Genotype } & \multirow[t]{2}{*}{ IS(n) } & \multirow[t]{2}{*}{ Pearson year(y) } & \multirow[t]{2}{*}{ Incident density $\left(/ 10^{4}\right)$} & \multicolumn{3}{|c|}{ HR $(95 \% \mathrm{CI}), P$ value } \\
\hline & & & & & & Additive model & Dominant model & Recessive model \\
\hline \multirow[t]{9}{*}{ rs2268350 } & \multirow[t]{3}{*}{ Male } & $\mathrm{CC}$ & 30 & 3425.41 & 87.58 & $1.356(0.989-1.858)$ & $1.152(0.733-1.811)$ & $2.057(1.24-3.411)$ \\
\hline & & CT & 44 & 4058.09 & 108.43 & $P=0.058$ & $P=0.539$ & $P=0.005$ \\
\hline & & $\mathrm{TT}$ & 20 & 1156.86 & 172.88 & & & \\
\hline & \multirow[t]{3}{*}{ Smoking } & $\mathrm{CC}$ & 14 & 2097.28 & 66.75 & $1.767(1.125-2.778)$ & $1.52(0.781-2.959)$ & $2.933(1.464-5.875)$ \\
\hline & & $\mathrm{CT}$ & 21 & 2374.57 & 88.44 & $P=0.014$ & $P=0.217$ & $P=0.002$ \\
\hline & & $\mathrm{TT}$ & 12 & 741.86 & 161.76 & & & \\
\hline & \multirow[t]{3}{*}{ Drinking } & $\mathrm{CC}$ & 9 & 1909.32 & 47.14 & $1.855(1.119-3.074)$ & 2.942(1.235-7.007) & $1.69(0.684-4.175)$ \\
\hline & & CT & 23 & 2113.95 & 108.8 & $P=0.017$ & $P=0.015$ & $P=0.256$ \\
\hline & & TT & 6 & 640.57 & 93.67 & & & \\
\hline \multirow[t]{6}{*}{ rs2672587 } & \multirow[t]{3}{*}{ Non-smoking } & $\mathrm{CC}$ & 26 & 4160.35 & 62.49 & $1.149(0.906-1.457)$ & $1.662(1.076-2.569)$ & $0.901(0.599-1.357)$ \\
\hline & & CG & 84 & 7966.11 & 105.45 & $P=0.253$ & $P=0.022$ & $P=0.619$ \\
\hline & & GG & 30 & 3769.38 & 79.59 & & & \\
\hline & \multirow[t]{3}{*}{ Smoking } & $\mathrm{CC}$ & 18 & 1368.69 & 131.51 & $0.631(0.405-0.985)$ & $0.608(0.324-1.141)$ & $0.436(0.171-1.112)$ \\
\hline & & CG & 23 & 2648.71 & 86.83 & $P=0.043$ & $P=0.121$ & $P=0.082$ \\
\hline & & GG & 6 & 1196.32 & 50.15 & & & \\
\hline \multirow[t]{9}{*}{ rs3793917 } & \multirow[t]{3}{*}{ Non-smoking } & $\mathrm{CC}$ & 28 & 4785.95 & 58.5 & $1.328(1.053-1.675)$ & $2.080(1.366-3.167)$ & $1.049(0.693-1.588)$ \\
\hline & & CG & 83 & 7890.11 & 105.19 & $P=0.016$ & $P=0.001$ & $P=0.819$ \\
\hline & & GG & 29 & 3207.34 & 90.42 & & & \\
\hline & \multirow[t]{3}{*}{ Smoking } & $\mathrm{CC}$ & 21 & 1590.4 & 132.04 & $0.498(0.312-0.796)$ & $0.452(0.244-0.837)$ & $0.302(0.100-0.911)$ \\
\hline & & CG & 21 & 2623.94 & 80.03 & $P=0.004$ & $P=0.011$ & $P=0.033$ \\
\hline & & GG & 5 & 999.38 & 50.03 & & & \\
\hline & \multirow[t]{3}{*}{ Non-drinking } & $\mathrm{CC}$ & 35 & 4932.98 & 70.95 & $1.178(0.94-1.476)$ & $1.647(1.120-2.420)$ & $0.91(0.6-1.381)$ \\
\hline & & CG & 86 & 8204.34 & 104.82 & $P=0.154$ & $P=0.011$ & $P=0.657$ \\
\hline & & GG & 28 & 3297.3 & 84.92 & & & \\
\hline
\end{tabular}

Gender stratification adjusted for age, TCH, TG, LDL-C, LDL-C, BMI, drinking, smoking, HTN and T2DM. Smoking stratification adjusted for age, gender, TCH, TG, LDL-C, HDL-C, BMI, drinking, HTN and T2DM. Drinking stratification adjusted for age, gender, TCH, TG, LDL-C, HDL-C, BMI, smoking, HTN and T2DM.

In the non-smoking population, rs3793917 CG/GG carriers had an increased risk of IS than CC carriers, the adjusted HR $(95 \% \mathrm{CI})$ was 2.080 (1.366-3.167), $P=0.001$. The FDR-adjusted $P$ value was 0.048 . While in the smoking population, the variants of rs3793917 presented a significant additive effect on the decreased risk of IS, the adjusted HRs (95\% CIs) were $0.452(0.244-0.837)$ with $P$ value of 0.004 (Table 2). The association of rs3793917 (CG/GG vs. CC) with IS was also significant in non-drinking population [adjusted HR (95\% CI) $=1.647$ (1.120-2.420), P=0.011] whereas not significant in drinking population (Supplementary Table S3).

\section{Interaction analysis}

Additive interaction analysis showed that additive interaction between HTRA1 rs2268350 and smoking was statistically significant, after adjustment for age, gender, TC, TG, HDL, LDL, drinking, BMI, T2DM and HTN. The RERI, AP and S values $(95 \%$ CIs) were 0.271 (-0.071-0.613), 0.169 (0.030-0.308), 1.805 (1.009-3.229) and $P$ values were $0.120,0.017$ and 0.047 respectively (Table 3 ). All the data of other interaction analysis were listed in Supplementary Table S4.

Furthermore, the multiplicative interaction rs2268350 and smoking was also verified to be significant and adjusted HR $(95 \% \mathrm{CI})$ was 1.179 (1.013-1.372) with $P$ of 0.033 after adjustment for covariates.

\section{Modification effect analysis}

For the rs2268350 CC genotype carriers, drinkers had a decreased risk of ischemic stroke than non-drinkers, the HR $(95 \% \mathrm{CI})$ was 0.285 (0.110-0.734) and $P$ value was 0.009 , after adjustment for age, gender, TC, TG, HDL, LDL, smoking, BMI, T2DM and HTN. The $P$ value of heterogeneity test was 0.005 after adjustments for covariates. For the rs2268350 TT genotype, females had an increased risk of ischemic stroke than males after adjustment for other confounding factors, the adjusted $\mathrm{HR}(95 \% \mathrm{CI})$ was 0.379 (0.144-0.998) and $P$ value was 0.049 .

According to those who carried CC genotype of rs3793917, smokers had an increased risk of ischemic stroke than CG and GG genotype carriers after adjustment for the other confounding factors, the adjusted HR (95\%CI) was 7.503 (2.624-21.457) and $P$ value was $0.171 \times 10^{-3}$. The $P$ value for heterogeneity test was less than 0.001 after adjustments for covariates among smokers carrying CC, CG and GG genotypes of rs3793917 (Table 3).

As for carriers with GG genotype of rs2672587, smokers had an increased risk of ischemic stroke than CC and CG genotype carriers, after adjustment for the remaining confounding factors, the adjusted HR (95\%CI) was 4.821 (1.526-15.229) with $P$ value of 0.007 . The $P$ value for heterogeneity test was 0.017 after adjustments for covariates among smokers carrying CC, CG and GG genotypes of rs2672587 (Table 4). 
Table 3. Additive interaction analysis of gender, smoking, drinking and HTRAI polymorphisms on IS incidence

\begin{tabular}{|c|c|c|c|c|c|c|c|c|c|}
\hline SNPs & Modified factor & Genotype & Stratum & IS(n) & Adjusted HR $(95 \% \mathrm{CI})$ & Adjusted $P$ & Adjusted RERI & Adjusted AP & Adjusted S \\
\hline \multirow[t]{12}{*}{ rs2268350 } & Gender & $\mathrm{CC}$ & Male & 30 & 1.00(reference) & & & & \\
\hline & & $\mathrm{CC}$ & Female & 35 & $0.739(0.424-1.286)$ & 0.284 & $0.013(-0.132-0.157)$ & $0.013(-0.132-0.158)$ & $0.661(0.000-5979.913)$ \\
\hline & & $\mathrm{CT}+\mathrm{TT}$ & Male & 64 & $1.081(0.691-1.693)$ & 0.733 & $P=0.863$ & $P=0.861$ & $P=0.929$ \\
\hline & & $\mathrm{CT}+\mathrm{TT}$ & Female & 58 & $0.849(0.508-1.419)$ & 0.532 & & & \\
\hline & Smoking & CC & Non-smoking & 51 & 1.00 (reference) & & & & \\
\hline & & CC & Smoking & 14 & $0.904(0.457-1.792)$ & 0.773 & $0.271(-0.071-0.613)$ & $0.169(0.030-0.308)$ & $1.805(1.009-3.229)$ \\
\hline & & $\mathrm{CT}+\mathrm{TT}$ & Non-smoking & 89 & $1.028(0.723-1.461)$ & 0.879 & $P=0.120$ & $P=0.017$ & $P=0.047$ \\
\hline & & $\mathrm{CT}+\mathrm{TT}$ & Smoking & 33 & $1.315(0.778-2.225)$ & 0.307 & & & \\
\hline & Drinking & $\mathrm{CC}$ & Non-drinking & 56 & 1.00 (reference) & & & & \\
\hline & & $\mathrm{CC}$ & Drinking & 9 & $0.385(0.174-0.855)$ & 0.019 & $0.064(-0.1517-0.2805)$ & $0.065(-0.135-0.269)$ & $0.015\left(0.000-1.494^{*} 10^{122}\right)$ \\
\hline & & $\mathrm{CT}+\mathrm{TT}$ & Non-drinking & 93 & $0.949(0.676-1.331)$ & 0.762 & $P=0.559$ & $P=0.526$ & $P=0.977$ \\
\hline & & $\mathrm{CT}+\mathrm{TT}$ & Drinking & 29 & $0.899(0.536-1.508)$ & 0.686 & & & \\
\hline \multirow[t]{4}{*}{ rs2672587 } & Smoking & $\mathrm{CC}$ & Non-smoking & 26 & 1.00(reference) & & & & \\
\hline & & $\mathrm{CC}$ & Smoking & 18 & $2.361(1.212-4.600)$ & 0.012 & $-0.061(-0.234-0.111)$ & $-0.057(-0.222-0.108)$ & $0.559(0.0484-6.453)$ \\
\hline & & $\mathrm{CG}+\mathrm{GG}$ & Non-smoking & 114 & $1.686(1.092-2.602)$ & 0.018 & $P=0.484$ & $P=0.498$ & $P=0.641$ \\
\hline & & CG+GG & Smoking & 29 & $1.463(0.792-2.701)$ & 0.224 & & & \\
\hline \multirow[t]{8}{*}{ rs3793917 } & Smoking & $\mathrm{CC}$ & Non-smoking & 28 & 1.00(reference) & & & & \\
\hline & & $\mathrm{CC}$ & Smoking & 21 & $3.211(1.704-6.051)$ & $3.06 \times 10^{-4}$ & $-0.0316(-0.21-0.147)$ & $-0.027(0.182-0.128)$ & $0.849(0.305-2.362)$ \\
\hline & & $\mathrm{CG}+\mathrm{GG}$ & Non-smoking & 112 & $2.061(1.355-3.135)$ & 0.001 & $P=0.728$ & $P=0.735$ & $P=0.754$ \\
\hline & & $\mathrm{CG}+\mathrm{GG}$ & Smoking & 26 & $1.456(0.786-2.696)$ & 0.232 & & & \\
\hline & Drinking & $\mathrm{CC}$ & Non-drinking & 35 & 1.00 (reference) & & & & \\
\hline & & $\mathrm{CC}$ & Drinking & 14 & $1.339(0.680-2.640)$ & 0.398 & $-0.047(-0.18-0.086)$ & $-0.063(-0.257-0.13)$ & $1.227(0.749-2.008)$ \\
\hline & & $C G+G G$ & Non-drinking & 114 & $1.608(1.095-2.360)$ & 0.015 & $P=0.487$ & $P=0.522$ & $P=0.416$ \\
\hline & & $C G+G G$ & Drinking & 24 & $0.913(0.509-1.639)$ & 0.76 & & & \\
\hline
\end{tabular}

Gender stratification adjusted for age, TCH, TG, LDL-C, LDL-C, BMI, drinking, smoking, HTN and T2DM. Smoking stratification adjusted for age, gender, TCH, TG, LDL-C, HDL-C, BMI, drinking, HTN and T2DM. Drinking stratification adjusted for age, gender, TCH, TG, LDL-C, HDL-C, BMI, smoking, HTN and T2DM.

Table 4. Association analysis for the modification effect of gender, smoking and drinking on IS incidence

\begin{tabular}{|c|c|c|c|c|c|c|c|c|c|}
\hline SNP & Genotype & Modification factor & IS(n) & HR $(95 \% \mathrm{CI})$ & $P$ & Heterogeneity test ${ }^{\mathrm{b}}$ & $\mathrm{HR}(95 \% \mathrm{CI})^{\mathrm{a}}$ & $\mathrm{Pa}$ & Heterogeneity test $t^{b}$ \\
\hline \multirow[t]{9}{*}{ rs2268350 } & $\mathrm{CC}$ & \multirow[t]{3}{*}{ Smoking } & 65 & $0.678(0.366-1.255)$ & 0.216 & \multirow[t]{3}{*}{$P=0.124$} & $1.043(0.483-2.255)$ & 0.915 & \multirow[t]{3}{*}{$P=0.347$} \\
\hline & CT & & 88 & $0.978(0.596-1.606)$ & 0.931 & & $0.943(0.485-1.833)$ & 0.863 & \\
\hline & TT & & 34 & $1.819(0.883-3.747)$ & 0.105 & & $2.210(0.831-5.873)$ & 0.112 & \\
\hline & $\mathrm{CC}$ & \multirow[t]{3}{*}{ Drinking } & 65 & $0.329(0.141-0.768)$ & 0.010 & \multirow[t]{3}{*}{$P=0.037$} & $0.285(0.110-0.734)$ & 0.009 & \multirow[t]{3}{*}{$P=0.005$} \\
\hline & СТ & & 88 & $1.177(0.727-1.905)$ & 0.508 & & $1.468(0.811-2.657)$ & 0.205 & \\
\hline & TT & & 34 & $0.822(0.337-2.005)$ & 0.667 & & $0.368(0.133-1.019)$ & 0.054 & \\
\hline & $\mathrm{CC}$ & \multirow[t]{3}{*}{ Gender } & 65 & $0.955(0.576-1.582)$ & 0.858 & \multirow[t]{3}{*}{$P=0.259$} & $0.547(0.296-1.013)$ & 0.055 & \multirow[t]{3}{*}{$P=0.105$} \\
\hline & $\mathrm{CT}$ & & 88 & $0.841(0.548-1.291)$ & 0.429 & & $1.091(0.618-1.925)$ & 0.765 & \\
\hline & TT & & 34 & $0.475(0.238-0.950)$ & 0.035 & & $0.379(0.144-0.998)$ & 0.049 & \\
\hline \multirow[t]{6}{*}{ rs3793917 } & $\mathrm{CC}$ & \multirow[t]{3}{*}{ Smoking } & 49 & $2.355(1.316-4.213)$ & 0.004 & \multirow[t]{3}{*}{$P=0.002$} & 7.503(2.624-21.457) & $0.171 \times 10^{-3}$ & \multirow[t]{3}{*}{$P<0.001$} \\
\hline & CG & & 104 & $0.750(0.463-1.213)$ & 0.241 & & $0.659(0.359-1.211)$ & 0.18 & \\
\hline & GG & & 34 & $0.397(0.139-1.135)$ & 0.085 & & $0.547(0.156-1.912)$ & 0.3445 & \\
\hline & $\mathrm{CC}$ & \multirow[t]{3}{*}{ Drinking } & 49 & $0.974(0.496-1.912)$ & 0.94 & \multirow[t]{3}{*}{$P=0.446$} & $0.635(0.280-1.440)$ & 0.277 & \multirow[t]{3}{*}{$P=0.967$} \\
\hline & CG & & 104 & $0.720(0.432-1.200)$ & 0.207 & & $0.727(0.392-1.347)$ & 0.311 & \\
\hline & GG & & 34 & $0.435(0.151-1.253)$ & 0.123 & & $0.689(0.203-2.340)$ & 0.551 & \\
\hline \multirow[t]{3}{*}{ rs2672587 } & $\mathrm{CC}$ & \multirow[t]{3}{*}{ Smoking } & 44 & $2.076(1.123-3.837)$ & 0.020 & \multirow[t]{3}{*}{$P=0.015$} & $4.821(1.526-15.229)$ & 0.007 & \multirow[t]{3}{*}{$P=0.017$} \\
\hline & CG & & 107 & $0.819(0.514-1.306)$ & 0.402 & & $0.846(0.464-1.539)$ & 0.583 & \\
\hline & GG & & 36 & $0.483(0.187-1.253)$ & 0.135 & & $0.616(0.204-1.860)$ & 0.391 & \\
\hline
\end{tabular}

a. Adjusted for age, gender, TC, TG, HDL, LDL, BMI, drinking, smoking, HTN and T2DM. b. $P$ value of the heterogeneity test based on the $\chi^{2}$ based on $Q$ test.

\section{mRNA expression levels comparing between IS cases and controls}

The demographic and clinical characteristics for the case-control study of ischemic stroke were listed in Supplementary Table S5. The mRNA expression levels $(2-\Delta \Delta \mathrm{CT})$ were $1.344(0.759,2.452)$ and 1.112 $(0.439,2.379)$ in IS cases and controls respectively while no statistical significance was detected (Figure S1), $\mathrm{Z}=0.944, \mathrm{P}=0.345$.

In the whole sample of IS cases and controls, smokers had a relative lower HTRA1 mRNA levels of
$0.966(0.451,1.805)$ compared with non-smokers 1.402 $(0.609,2.498)$ and the $P$ value was 0.049 . Whereas, there was not the similar difference in IS cases or controls separately.

A significant difference of the mRNA expression levels $\left(2^{-\Delta \Delta C T}\right)$ was found between CC [1.590 (0.945, $2.481)]$ and CT/TT $[0.997(0.375,1.982)]$ genotypes of rs2268350 in IS cases with $P$ value of 0.044 but not in controls $(P>0.05)$. In addition, the mRNA level $\left(2^{-\Delta \Delta C T}\right)$ of HTRA1 in non-smokers with rs2268350 CC [1.647 $(1.137,2.493)]$ was significantly higher than smokers 
with rs2268350 CT/TT $[0.686(0.160,1.676)](\mathrm{P}=0.046)$ in IS cases (Figure 1). All the mRNA levels $\left(2^{-\Delta \Delta C T}\right)$ of different genotypes for each tagSNPs were listed in Supplementary Table S6.

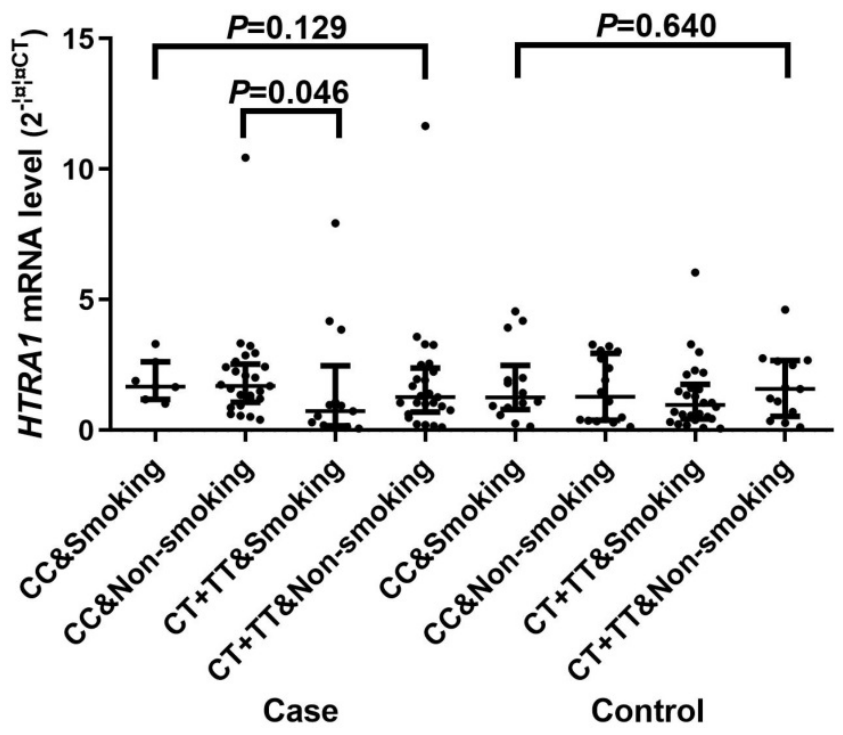

Figure 1. Comparisons of HTRAI mRNA levels among smokers with rs $2268350 \mathrm{CC}$, non-smokers with rs $2268350 \mathrm{CC}$, smokers with rs $2268350 \mathrm{CT} / \mathrm{TT}$ and non-smokers with rs $2268350 \mathrm{CT} / \mathrm{TT}$ in IS case group and control group respectively.

\section{Discussion}

In this prospective cohort study of Chinese population, we verified the association of HtrA1 and IS and identified significant interaction of rs2268350 $(\mathrm{C}>\mathrm{T})$ and smoking as well as differential mRNA expression level in PBMCs in IS cases and HTN controls. Notably, both the additive and multiplicative interaction between HTRA1 rs2268350 and smoking were to be found statistically significant, and the interaction might induce the development of IS probably through suppressing the HTRA1 mRNA expression in PBMCs. In addition, smoking devotes a two-fold increased risk of rs2672587 and rs3793917 CC genotypes on IS and even more after adjustment for covariates. These findings would further deepen our understanding of the role of gene-environmental interaction in the molecular mechanism of IS.

Cigarette smoke, which contains numerous potential oxidants is associated with the increased blood pressure level and cardiovascular disease mortality [30]. Vitro and in vivo studies indicated that the cigarette smoke components exerted their deleterious effects on DNA adducts via cytochrome P-450 (CYP)-dependent bioactivation [31] and inducing oxidative stress [32] and inflammation [33]. China now consumes about $40 \%$ of the world's total cigarettes consumption, especially in men [34]. Previous study has suggested that smoking could strengthen the HTRA1 additive effect on AMD [22]. Similar macular degeneration and cell apoptosis were also observed in patients with neovascular type (nAMD) [35], as well as stroke [36] and myocardial infarction (MI) [37]. Cigarette smoking also could modify the genetic effect of LOC387715 rs110490924 on polypoidal choroidal vasculopathy [38]. In addition, previous cohort study showed that the nAMD significantly increased the risk of stroke $(\mathrm{HR}=1.3)$, particularly hemorrhagic stroke $(\mathrm{HR}=1.70)[39]$. These findings would help to understand the molecular mechanisms how smoking affect stroke and promote tobacco control strategy in preventing the onset of stroke.

For CARASIL, the pathogenic mutations of HTRA1 were suggested to impair the regulatory function of HTRA1 and thus directly increase the level of TGF- $\beta 1$ in cerebral small arteries [21]. Accordingly, as a downstream effector of the TGF- $\beta 1$ signaling cascade in brain tissue, the phosphorylated SMAD protein expression levels significantly increased [18]. HTRA1 gene also regulates scar formation in the pathogenesis of scars through the activation of latent TGF- $\beta 1$ in keloid fibroblasts [40]. All these evidences suggest that HTRA1 participates in the pathogenesis of stroke by regulating angiogenesis via TGF- $\beta$ signaling.

Our most notable findings illustrated that the additive and multiplicative interactions between smoking and HTRA1 rs2268350 were both statistically significant on the incidence of IS. Furthermore, among carriers with CC genotype for rs3793917 and rs2672587, smokers always showed a higher risk of IS than non-smokers. Both smoking and the risk allele $\mathrm{T}$ of HTRA1 rs2268350 could suppress the HTRA1 mRNA expression. Therefore, smoking and the affective allele of HTRA1 together could be applied to forecast the risk of stroke. The further replication of this interaction would be warranted in other follow-up populations.

The main advantages of this study are that we firstly verified the association of HtrA1 with IS and the interactions between smoking and HTRA1 polymorphisms in a cohort study in Chinese population. Particularly, the results of differential HTRA1 mRNA expression further provided functional evidence of transcriptional level. This study also has the following limitations: firstly, we just selected and tested four tagSNPs of HtrA1 with MAF over 0.05 and that might miss some rare SNP as well as the biological effect might be presented by closely linked loci nearby. Secondly, we evaluated the interaction of smoking status at baseline and HtrA1 genetic variations, whereas we didn't check the change of smoking exposure during follow-up and 
this will prevent us from doing more accurate analysis instead of conventional analytical methods for cohort study. Lastly, although we adjusted the traditional confounders, the findings of this study warrant to be further validated by other studies refraining from potential selection bias.

In conclusion, the findings of this study support that HTRA1 harbor genetic variations that contribute to the susceptibility of IS in Chinese Han population and the population-based evidence of HTRA1 rs2268350 variations interacting with smoking, and HTRA1 mRNA differentially expressing among smoking status and rs2268350 genotypes in the patients with IS suggest that smoking modifies the genetic effect of HTRA1 on IS. Further investigation would be recommended into the exact mechanism of relevant gene-environment interaction.

\section{Supplementary Material}

Supplementary figures and tables. http://www.medsci.org/v18p1840s1.pdf

\section{Acknowledgements}

This work was supported by National Natural Science Foundation of China (No. 81573232 and No. 81273165), Jiangsu Provincial Fourth "333 Project" and the Priority Academic Program for the Development of Jiangsu Higher Education Institutions (Public Health and Preventive Medicine) and the Flagship Major Development of Jiangsu Higher Education Institutions. The funders had no role in study design, data collection and analysis, decision to publish, or preparation of the manuscript.

\section{Competing Interests}

The authors have declared that no competing interest exists.

\section{References}

1. Collaborators GBDCoD. Global, regional, and national age-sex specific mortality for 264 causes of death, 1980-2016: a systematic analysis for the Global Burden of Disease Study 2016. Lancet. 2017; 390: 1151-210.

2. Donnan GA, Fisher M, Macleod M, Davis SM. Stroke. Lancet. 2008; 371: 1612-23.

3. Chen Z, Jiang B, Ru X, Sun H, Sun D, Liu X, et al. Mortality of Stroke and Its Subtypes in China: Results from a Nationwide Population-Based Survey. Neuroepidemiology. 2017; 48: 95-102.

4. Yang G, Wang Y, Zeng Y, Gao GF, Liang X, Zhou M, et al. Rapid health transition in China, 1990-2010: findings from the Global Burden of Disease Study 2010. Lancet. 2013; 381: 1987-2015.

5. Thrift AG, Dewey HM, Macdonell RA, McNeil JJ, Donnan GA. Incidence of the major stroke subtypes: initial findings from the North East Melbourne stroke incidence study (NEMESIS). Stroke. 2001; 32: 1732-8.

6. Wang J, Wen X, Li W, Li X, Wang Y, Lu W. Risk Factors for Stroke in the Chinese Population: A Systematic Review and Meta-Analysis. Journal of stroke and cerebrovascular diseases : the official journal of National Stroke Association. 2017; 26: 509-17.

7. O'Donnell MJ, Xavier D, Liu L, Zhang H, Chin SL, Rao-Melacini P, et al. Risk factors for ischaemic and intracerebral haemorrhagic stroke in 22 countries (the INTERSTROKE study): a case-control study. Lancet. 2010; 376: 112-23.

8. Mutlu U, Swanson SA, Klaver CCW, Hofman A, Koudstaal PJ, Ikram MA, et al. The mediating role of the venules between smoking and ischemic stroke. European journal of epidemiology. 2018.
9. McGeechan K, Liew G, Macaskill P, Irwig L, Klein R, Klein BE, et al. Prediction of incident stroke events based on retinal vessel caliber: a systematic review and individual-participant meta-analysis. American journal of epidemiology. 2009; 170: 1323-32.

10. Markidan J, Cole JW, Cronin CA, Merino JG, Phipps MS, Wozniak MA, et al. Smoking and Risk of Ischemic Stroke in Young Men. Stroke. 2018; 49: 1276-8.

11. Fischer F, Kraemer A. Meta-analysis of the association between second-hand smoke exposure and ischaemic heart diseases, COPD and stroke. BMC public health. 2015; 15: 1202.

12. Sun ML, Sun LM, Wang YQ. Peptide selectivity between the PDZ domains of human pregnancy-related serine proteases (HtrA1, HtrA2, HtrA3, and HtrA4) can be reshaped by different halogen probes. Journal of molecular recognition : JMR. 2017.

13. Zurawa-Janicka D, Wenta T, Jarzab M, Skorko-Glonek J, Glaza P, Gieldon A, et al. Structural insights into the activation mechanisms of human HtrA serine proteases. Archives of biochemistry and biophysics. 2017; 621: 6-23.

14. Chien J, Ota T, Aletti G, Shridhar R, Boccellino M, Quagliuolo L, et al. Serine protease HtrA1 associates with microtubules and inhibits cell migration. Molecular and cellular biology. 2009; 29: 4177-87.

15. $\mathrm{Yu} T$, Chen $\mathrm{CZ}$, Xing $\mathrm{YQ}$. Inhibition of cell proliferation, migration and apoptosis in blue-light illuminated human retinal pigment epithelium cells by down-regulation of HtrA1. International journal of ophthalmology. 2017; 10: 524-9.

16. Oka C, Tsujimoto R, Kajikawa M, Koshiba-Takeuchi K, Ina J, Yano M, et al. HtrA1 serine protease inhibits signaling mediated by Tgfbeta family proteins. Development. 2004; 131: 1041-53.

17. Beaufort N, Scharrer E, Kremmer E, Lux V, Ehrmann M, Huber R, et al. Cerebral small vessel disease-related protease HtrA1 processes latent TGF-beta binding protein 1 and facilitates TGF-beta signaling. Proceedings of the National Academy of Sciences of the United States of America. 2014; 111: 16496-501.

18. Zhang L, Lim SL, Du H, Zhang M, Kozak I, Hannum G, et al. High temperature requirement factor A1 (HTRA1) gene regulates angiogenesis through transforming growth factor-beta family member growth differentiation factor 6 . The Journal of biological chemistry. 2012; 287: 1520-6.

19. Chien J, Campioni M, Shridhar V, Baldi A. HtrA serine proteases as potential therapeutic targets in cancer. Current cancer drug targets. 2009; 9: 451-68.

20. Jones A, Kumar S, Zhang N, Tong Z, Yang JH, Watt C, et al. Increased expression of multifunctional serine protease, HTRA1, in retinal pigment epithelium induces polypoidal choroidal vasculopathy in mice. Proceedings of the National Academy of Sciences of the United States of America. 2011; 108: 14578-83.

21. Hara K, Shiga A, Fukutake T, Nozaki H, Miyashita A, Yokoseki A, et al. Association of HTRA1 mutations and familial ischemic cerebral small-vessel disease. The New England journal of medicine. 2009; 360: 1729-39.

22. Tam PO, Ng TK, Liu DT, Chan WM, Chiang SW, Chen LJ, et al. HTRA1 variants in exudative age-related macular degeneration and interactions with smoking and CFH. Investigative ophthalmology \& visual science. 2008; 49: 2357-65

23. Tan RYY, Traylor M, Megy K, Duarte D, Deevi SVV, Shamardina O, et al. How common are single gene mutations as a cause for lacunar stroke? A targeted gene panel study. Neurology. 2019; 93: e2007-e20.

24. Nozaki H. [Consideration of the pathogenesis of CARASIL]. Rinsho shinkeigaku = Clinical neurology. 2012; 52: 1360-2.

25. Uemura $M$, Nozaki $H$, Onodera O. [Cerebral Autosomal Recessive Arteriopathy with Subcortical Infarcts and Leukoencephalopathy (CARASIL)]. Brain and nerve = Shinkei kenkyu no shinpo. 2017; 69: 25-33.

26. Shiga A, Nozaki H, Nishizawa M, Onodera O. [Molecular pathogenesis of cerebral autosomal recessive arteriopathy with subcortical infarcts and leukoencephalopathy]. Brain and nerve $=$ Shinkei kenkyu no shinpo. 2010; 62: 595-9.

27. Rannikmae K, Sivakumaran V, Millar H, Malik R, Anderson CD, Chong M, et al. COL4A2 is associated with lacunar ischemic stroke and deep ICH: Meta-analyses among 21,500 cases and 40,600 controls. Neurology. 2017; 89: 1829-39.

28. Chen J, Zhao X, Wang H, Chen Y, Wang W, Zhou W, et al. Common variants in TGFBR2 and miR-518 genes are associated with hypertension in the Chinese population. Am J Hypertens. 2014; 27: 1268-76.

29. Benjamini Y, Hochberg Y. Controlling the false discovery rate: a practical and powerful approach to multiple testing. Journal of the Royal Statistical Society Series B. 1995; 57: 2.

30. Honjo $\mathrm{K}$, Iso $\mathrm{H}$, Tsugane $\mathrm{S}$, Tamakoshi $\mathrm{A}$, Satoh $\mathrm{H}$, Tajima $\mathrm{K}$, et al. The effects of smoking and smoking cessation on mortality from cardiovascular disease among Japanese: pooled analysis of three large-scale cohort studies in Japan. Tobacco control. 2010; 19: 50-7.

31. Hecht SS. Cigarette smoking and lung cancer: chemical mechanisms and approaches to prevention. The Lancet Oncology. 2002; 3: 461-9.

32. Gebel S, Gerstmayer B, Bosio A, Haussmann HJ, Van Miert E, Muller T. Gene expression profiling in respiratory tissues from rats exposed to mainstream cigarette smoke. Carcinogenesis. 2004; 25: 169-78

33. Ambrose JA, Barua RS. The pathophysiology of cigarette smoking and cardiovascular disease: an update. Journal of the American College of Cardiology. 2004; 43: 1731-7.

34. Yang G, Wang Y, Wu Y, Yang J, Wan X. The road to effective tobacco control in China. Lancet. 2015; 385: 1019-28. 
35. Whitmore SS, Sohn EH, Chirco KR, Drack AV, Stone EM, Tucker BA, et al. Complement activation and choriocapillaris loss in early AMD: implications for pathophysiology and therapy. Progress in retinal and eye research. 2015; 45: 1-29.

36. Yan T, Chopp M, Chen J. Experimental animal models and inflammatory cellular changes in cerebral ischemic and hemorrhagic stroke. Neuroscience bulletin. 2015; 31: 717-34

37. Wu J, Stefaniak J, Hafner C, Schramel JP, Kaun C, Wojta J, et al. Intermittent Hypoxia Causes Inflammation and Injury to Human Adult Cardiac Myocytes. Anesthesia and analgesia. 2016; 122: 373-80.

38. Nakanishi H, Yamashiro K, Yamada R, Gotoh N, Hayashi H, Nakata I, et al. Joint effect of cigarette smoking and CFH and LOC387715/HTRA1 polymorphisms on polypoidal choroidal vasculopathy. Investigative ophthalmology \& visual science. 2010; 51: 6183-7.

39. Lee WA, Cheng CL, Lee CH, Kao Yang YH, Lin SJ, Hsieh CY. Risks of newly onset hemorrhagic stroke in patients with neovascular age-related macular degeneration. Pharmacoepidemiology and drug safety. 2017; 26: 1277-85.

40. Jin SF, Wang $\mathrm{YX}, \mathrm{Xu} N$, Sun $\mathrm{Q}$, Wang $\mathrm{CC}$, Lv MZ, et al. High temperature requirement factor A1 (HTRA1) regulates the activation of latent TGF-beta1 in keloid fibroblasts. Cellular and molecular biology. 2018; 64: 107-10. 